\title{
Management of Venous Malformations
}

\author{
Michael Acord, MD ${ }^{1}$ Abhay Srinivasan, $\mathrm{MD}^{1}$ \\ ${ }^{1}$ Department of Radiology, Children's Hospital of Philadelphia, \\ Perelman School of Medicine at the University of Pennsylvania, \\ Philadelphia, Pennsylvania
}

\begin{abstract}
Address for correspondence Abhay Srinivasan, MD, Department of Radiology, Children's Hospital of Philadelphia, 3401 Civic Center Blvd, Philadelphia, PA 19104 (e-mail: srinivasaa@chop.edu).
\end{abstract}

Semin Intervent Radiol 2021;38:215-225

\begin{abstract}
Keywords

- venous malformation

- sclerotherapy

- pediatric

- interventional radiology

- embolization

Venous malformations (VMs) are slow-flow, congenital vascular anomalies that are a result of vascular dysgenesis. Clinical presentation and morbidity depend on size, location, and association with other syndromes. VMs are the most common symptomatic vascular anomaly referred for intervention, usually due to thrombosis, swelling, mass effect, functional compromise, or cosmetic concerns. Treatment for larger lesions can be challenging and a multidisciplinary approach involving medical, interventional, and surgical input is critical for comprehensive care. This article will assist the interventional radiologist in patient assessment and will discuss current techniques for treatment, means to minimize adverse events, and expected outcomes.
\end{abstract}

The 2018 International Society for the Study of Vascular Anomalies classification divides vascular malformations into four categories: simple, combined, of major vessels, and those associated with other anomalies. ${ }^{1}$ Of these, simple venous malformations (VMs) are the most commonly encountered in clinical practice and account for the majority of referrals to a vascular anomaly clinic for symptom management. ${ }^{2}$ Treatment can be challenging given the wide range of presentations, associated anomalies, and variations in potential approaches. This article will focus on the clinical and imaging assessment, indications for treatment, and means of intervention that should be important to consider both during clinical consultation and intervention.

\section{Pathogenesis and Clinical Presentation}

VMs are a result of an inborn error in vascular embryogenesis leading to the formation of abnormal endothelium and, as a result, thin-walled, nonfunctional, and ectatic veins. Greater than $90 \%$ of VMs are a result of a sporadic mutation, with the majority of phenotypes arising from endothelial dysgenesis via the TIE2-PI3K (phosphoinositol-3-kinase)-AKT-mTOR (mammalian target of rapamycin) pathway ( - Fig. 1). ${ }^{3-5}$ A gain-offunction somatic mutation in TEK leads to the overexpression of receptor tyrosine kinase TIE2, which is expressed on venous endothelial cells, resulting in dysplasia of the basement mem- brane and endothelium. While only seen in $25 \%$ of sporadic VMs, a somatic mutation in PIK3CA leads to unregulated activation of PI3K, which affects cell proliferation, migration, and survival. ${ }^{2}$ Mosaic mutations in this pathway are also the cause of VM-predominant overgrowth entities (known as the PIK3CA-related overgrowth spectrum or PROS), which include Klippel-Trenaunay syndrome (KTS) and CLOVES (congenital lipomatous overgrowth, vascular malformations, epidermal nevi, and spine/skeletal anomalies).

Present at birth, VMs generally grow commensurate with the patient, do not spontaneously regress, and can present at any age. Malformations due to sporadic mutations are usually unifocal while hereditary forms (such as glomuvenous malformation and, rarely, blue rubber bleb nevus/bean syndrome) are more commonly multifocal and are associated with other anomalies. Although VMs can be found at any location, approximately $40 \%$ occur in the head and neck. ${ }^{6}$ Rapid growth can be observed during teenage years and during pregnancy as sex hormones promote angiogenesis and proliferation. ${ }^{7}$ Increase in symptoms can also be seen in response to unrelated major trauma. When located superficially, they are seen as a blue, soft, and compressible mass that may be associated with varicosities. Documentation with clinical photographs is critical and is helpful when assessing treatment response. Unlike lymphatic malformations, due to increase venous blood flow, these lesions enlarge with Valsalva maneuvers or when placed in a
Issue Theme Interventional Radiology in Nonthrombotic Venous Interventions; Guest Editors, Ramona Gupta, MD and Ronald S. Winokur, MD (c) 2021. Thieme. All rights reserved. Thieme Medical Publishers, Inc., 333 Seventh Avenue, 18th Floor, New York, NY 10001, USA
DOI https://doi.org/ 10.1055/s-0041-1729743. ISSN 0739-9529. 


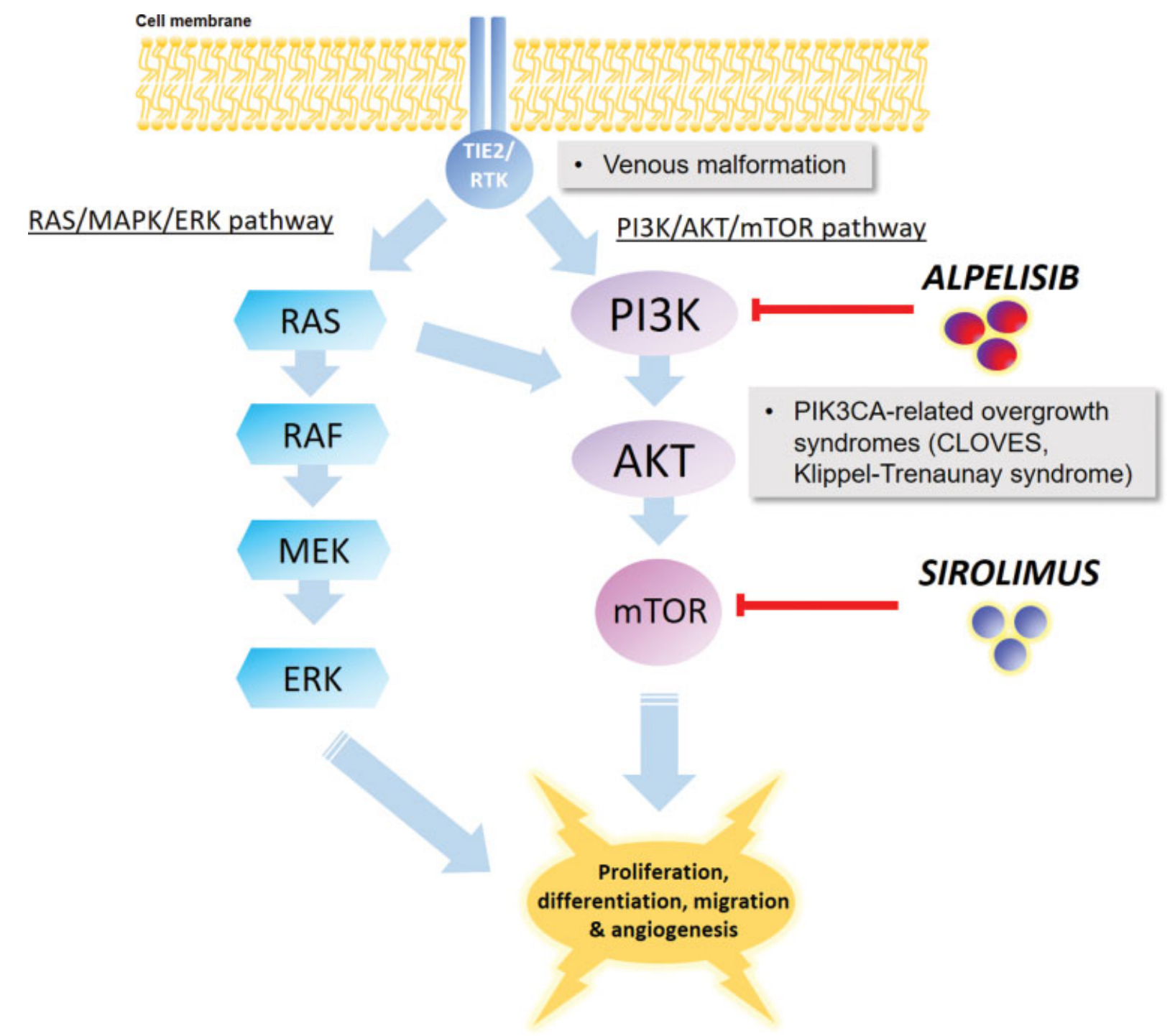

Fig. 1 Signal pathway involved in the pathogenesis of venous malformations and targets of systemic therapy. PI3K-AKT-mTOR signaling pathway and relevant vascular anomalies associated with each enzyme variant. Sirolimus inhibits mTOR. Alpelisib inhibits PI3K. AKT, AK strain transforming oncogene, or protein kinase B; CLOVES, congenital lipomatous overgrowth, vascular malformations, epidermal PIE 2/RTK, angiopoetin receptor and y60sine kinase, nevi, and spine/skeletal anomalies; ERK, extracellular receptor kinase; MEK, mitogen-activated protein kinase; mTOR, mammalian target of rapamycin; PI3K, phosphatidylinositol 3-kinase; PIK3CA, phosphatidylinositol 4,5-bisphosphate 3-kinase catalytic subunit $\alpha$; RAF, rapidly accelerated fibrosarcoma kinase; RAS, rat sarcoma oncogene.

dependent position. They should be cool to the touch and without pulsatility or thrill, unlike an arteriovenous malformation. Deeper lesions may not be evident in infancy and tend to present after puberty. In particular, lesions isolated to muscle are associated with later presentation and greater morbidity and exercise limitations. ${ }^{8}$ Complications include swelling and/or pain from distension, thrombosis or phlebitis, and joint pain from hemorrhage-related arthropathy with intra-articular involvement. ${ }^{9}$ When located in the extremities, these symptoms may increase with activity. Rarely, patients can present with pulmonary emboli in the setting of thrombosis if the malformation communicates with the deep venous system. ${ }^{10}$

\section{Patient Assessment}

After physical examination, imaging is performed for further characterization of VM and is also used to monitor treatment response. Ultrasound (US) is often the first screening modality at diagnosis. It is inexpensive, does not expose the patient to ionizing radiation, is readily available, and can be performed in the clinic. On gray-scale US, VMs appear as heterogeneously hypoechoic masses that are compressible. Tubular vascular channels may be observed, particularly with high-resolution imaging, but are not always visualized. Similarly, connections to normal venous drainage may not be evident. Phleboliths and thrombi, however, should be readily identified. On colorDoppler imaging, $84 \%$ of lesions will show flow, with the majority exhibiting monophasic, slow flow. ${ }^{11,12}$ When flow is extremely slow, it may not be detectable; thus, it is important to adjust the Doppler scale as low as possible to differentiate these lesions from lymphatic malformations.

Particularly in larger and deeper lesions, magnetic resonance imaging (MRI) is required for complete evaluation. Sequences for initial assessment must include a fat-suppressed 
T2-weighted sequence and a time-resolved angiographic sequence. The larger field-of-view allows assessment of transpatial and/or intra-articular extension, and involvement in critical and deeper structures, as well as assessment of the normal draining venous system. Computed tomography should not be routinely used unless there is need to characterize osseous involvement, which can manifest as osseous destruction or cortical thinning. On MRI, these are typically hypo- to isointense on T1-weighted sequences and are markedly hyperintense on T2-weighted sequences. Foci of hypointensity on T2-weighted imaging can be due to phleboliths or thrombi, which are confirmed by blooming artifact seen on gradient-echo sequences. While fluid-fluid levels are more commonly seen with lymphatic malformations, they can be present in larger VMs due to intralesional hemorrhage. Finally, VMs have varying degrees of delayed heterogenous enhancement and, depending on the timing between injection and imaging, some components may appear as nonenhancing. Some focal VMs have no or minimal egress, making them difficult to distinguish from lymphatic malformations.

With some extensive lesions, a planning venogram may be considered, as direct venography offers the best temporal and spatial resolution to assess the variable patterns of outflow seen with VM. For example, characterization of the deep venous system is critical prior to pursuing sclerotherapy in patients with extensive limb VMs, such as in KTS; diversion venography may be necessary, with placement of serial tourniquets over outflow veins. ${ }^{13}$ These are similar techniques which may be employed during sclerotherapy.

Prior to intervention, it is important to consider the differential diagnoses. Mimickers of VM in the pediatric population include vascular tumors, such as hemangiomas, which should exhibit a solid, soft-tissue component. Lesions such as peripheral nerve sheath tumors, congenital fibrosarcomas, and spindle cell hemangioendotheliomas have been mistaken for VM. ${ }^{14}$ Commonly mischaracterized as a VM, a fibroadipose vascular anomaly (FAVA) or phosphatase and tensin analog (PTEN) hamartoma can be differentiated by their fat content and tumor-like appearance on US.

As it is the stasis of blood and dysplastic endothelium that provoke thrombosis, extensive VM can harbor a localized intravascular coagulopathy (LIC). ${ }^{15}$ Sclerotherapy can progress LIC into disseminated intravascular coagulopathy (DIC), which is a medical emergency. ${ }^{16}$ Patients with larger VM are at greatest risk and it is recommended to evaluate for decreased fibrinogen and elevated D-dimer levels. If the patient is found to have LIC, then low-molecular-weight heparin (LMWH) is recommended prior to intervention or during pregnancy to prevent DIC. ${ }^{17,18}$ The use of a direct oral anticoagulant (DOAC), in lieu of LMWH injection, for DIC prophylaxis has also been described. ${ }^{19}$

\section{Treatment}

Clinical decision-making is best performed in an interventional radiology or multidisciplinary clinic, with review of all imaging. After accurate diagnosis, an individual treatment plan is created with a combination of therapies. It is important to discuss with the patient and family that the goal of intervention is not complete cure, but rather to decrease pain, the risk of future complications, and improve function. The need for staged or repeat treatment should also be discussed. A detailed pain history should be obtained, preferably with use of standardized pain questionnaires such as the Brief Pain Inventory or PedsQL. ${ }^{20,21}$

Available treatment modalities for VM include sclerotherapy, surgery (primary excision or excision after embolization), ablation, and oral pharmacotherapy. Systematic reviews have not established a benefit of sclerotherapy as an alternative to primary surgery, mainly due to limitations in study quality. ${ }^{22}$ However, due to its minimally invasive technique, sclerotherapy is currently the predominant modality in many centers. The Society of Interventional Radiology recommends preprocedure antibiotics for the treatment of vascular malformations, a position also endorsed by the Cardiovascular and Interventional Radiological Society of Europe. ${ }^{23}$

\section{Sclerotherapy}

\section{General Technique}

Effective sclerotherapy requires a comprehensive injury to the abnormal endothelium, and this relies on two concepts: dwell time and endothelial contact. Percutaneous sclerotherapy is performed by US-guided access of the lesion with acquisition of blood return, followed by meticulous fluoroscopic venography and injection of sclerosant. Small bore (21- or 22-gauge [G]) needles or sheathed needles (peripheral intravenous [IV] cannula) are commonly used, with short IV connection tubing. High-quality venography is crucial to safe and effective sclerotherapy, as the distribution, volume, and outflow of the malformation must be assessed. Based on outflow, VMs can be categorized into four types ( - Fig. 2). ${ }^{14}$ Outflow is characterized by venography and can be aided by preprocedure imaging, and determines the complexity of the treatment (-Fig. 3a-c). Control of outflow is central to sclerotherapy of VM, as this is necessary to increase dwell time, avoid nontarget sclerotherapy of normal veins and tissues, and
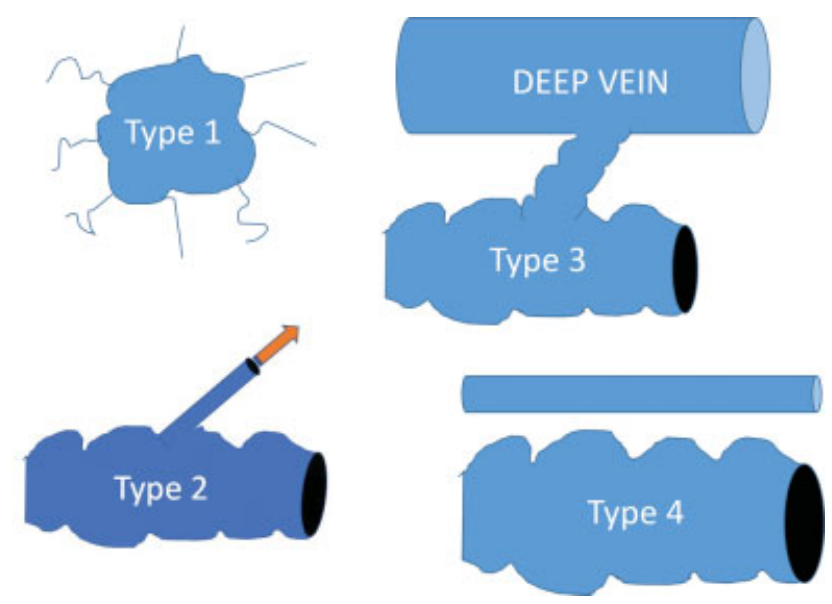

Fig. 2 Graphic depicting classification of venous malformation based on outflow. Counterclockwise, type 1 are isolated without drainage, type 2 drain into normal veins, type 3 drain into dysplastic veins that connect to the deep system, and type 4 are primarily ectatic veins. 

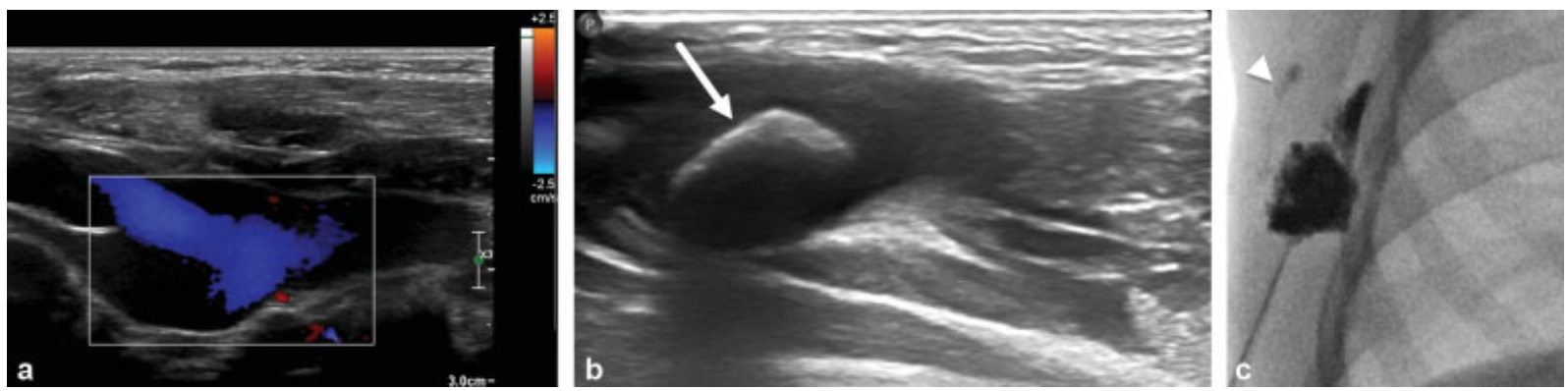

Fig. 3 A 4-year-old male with a type 2 venous malformation of the chest wall. (a) Ultrasound shows tubular, anechoic channels with slow flow and (b) linear echogenic, shadowing phlebolith (arrow). The latter is pathognomonic for a venous malformation. (c) Upon direct injection of the malformation, a sac-like structure overlying the ribs opacifies with contrast and shows drainage into a normal caliber vein (arrowhead). This was successfully treated with $5 \mathrm{~mL}$ STS foam.

reduce the risk of thromboembolism from malformations with a direct communication to the deep venous system.

It is also crucial that lesions never be forcefully injected or overfilled. Lesion volume and ease of injection are assessed on the venogram, and a volume of sclerosant reasonably commensurate to lesion volume must be injected. Adequate coverage of the lesion can be assessed with roadmap (subtracted) fluoroscopy. If the sclerosant is not radio-opaque, the lesion is first filled with contrast and then the contrast can be seen being displaced by the sclerosant with roadmap imaging. US, particularly when the sclerosant is foamed, is useful to assess lesion coverage. Aggressive injection of the lesion can result in extravasation or arterial reflux, and produce local tissue injury.

The double-needle technique is often necessary for effective sclerotherapy, and involves placing two or more open needles at opposite poles of the malformation ( - Fig. $\mathbf{4 a - c}$ ). The first is used to inject sclerosant until it is seen to egress through the other(s). The additional needle(s) allow a path of low resistance for sclerosant to permeate from one end of the lesion to the other, thus ensuring a more complete distribution. ${ }^{24}$ The double-needle technique may provide indirect outflow control, as egress can be directed away from undesirable outflow veins by appropriate needle positioning. For extremity VM with direct connection to the deep system, constant infusion of heparinized saline into the deep veins will also guard against sclerosant-induced injury to the deep veins.
Direct outflow control methods involve either temporary or permanent occlusion of draining veins. Often, a combination of these methods may be necessary, depending on the robustness of outflow and location of the lesion. Such methods include external compression (manually or by use of a sterile tourniquet, endovascular balloon occlusion, injection of glue, percutaneous ligation, and/or deployment of plugs or coils). ${ }^{25}$

Endovascular coils or plugs are commonly placed in large outflow veins with appropriate landing zones (- Fig. 5a-c). When sizing coils, it is important to be mindful of the fact that veins are distensible and may spasm during measurement or deployment. Any distension of the lumen after deployment may result in embolization; so, it is advisable to oversize by 30 to $50 \%$. While pushable or detachable coils may be used, the use of an initial detachable coil as a frame may be beneficial and provide some reassurance against coil embolization.

Endovascular balloon occlusion is most amenable for the treatment of type III VMs, with short and direct communication to deep veins. The deep veins are accessed separately from the malformation, and a balloon is positioned over a wire and inflated across the communication. Sclerotherapy is then performed (if necessary, with glue for more permanent outflow occlusion, as appropriate), and the balloon is deflated (-Fig. 6a, b).

Foamed sclerosants are commonly used in VM treatment because they promote both dwell time and physical contact with the endothelium. Foam, when adequately stable, is able
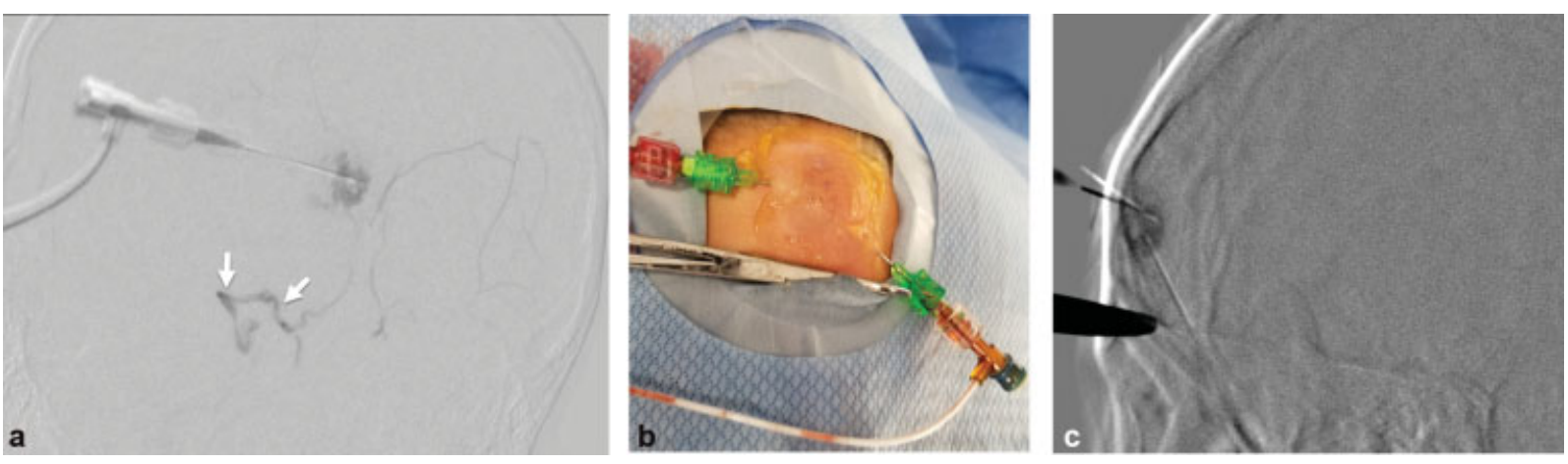

Fig. 4 An 11-year-old male with a type 2 venous malformation of the forehead treated using a double-needle technique. (a) Initial frontal venogram over the skull shows drainage into the superior ophthalmic vein (arrows). Egress of sclerosant was to be avoided. (b) Two 21 -gauge needles were inserted under ultrasound guidance for instillation of STS foam. Note a hemostat is used to control outflow. (c) Lateral fluoroscopic image of the skull shows successful injection of the radio-opaque sclerosant without communication to other veins or the dural venous sinuses. 

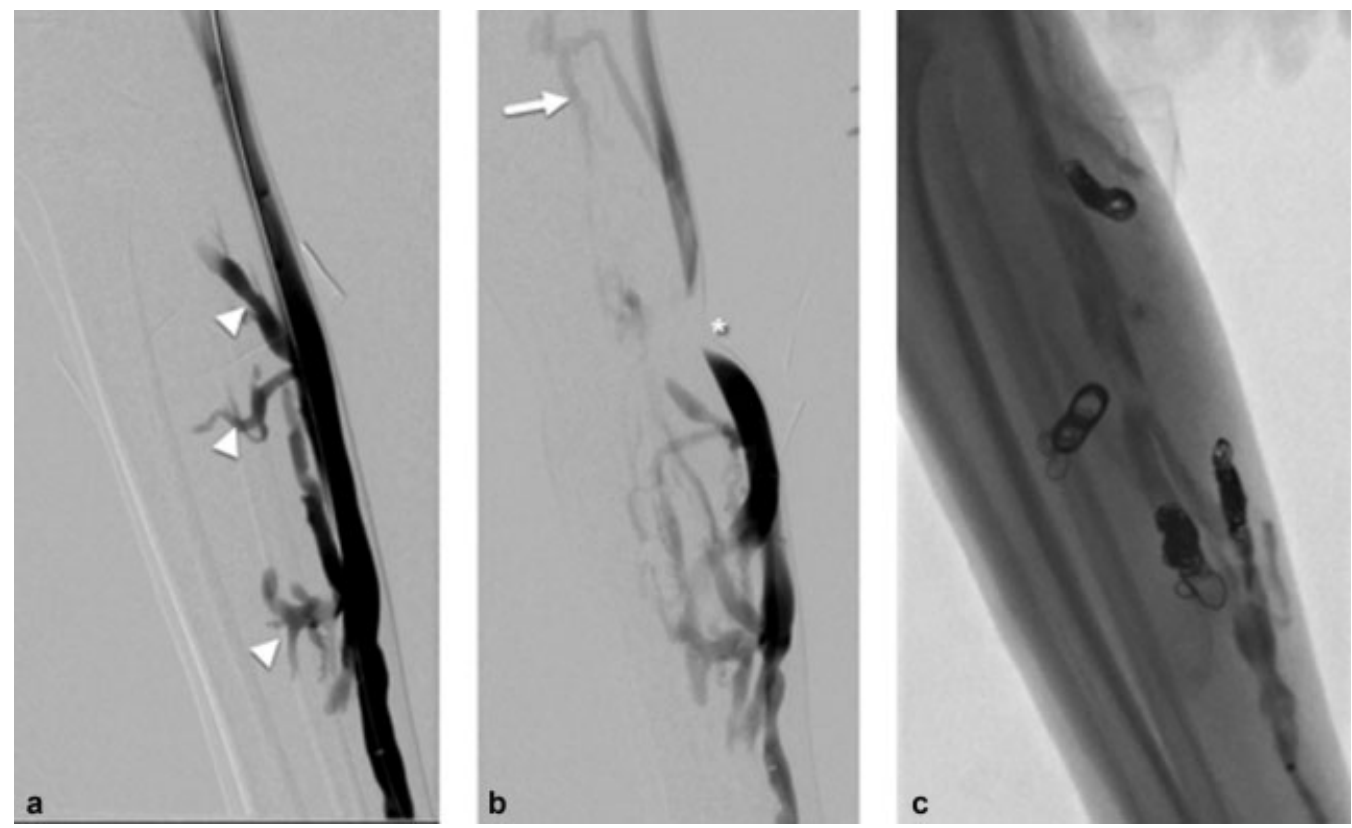

Fig. 5 A 10-year-old female with CLOVES syndrome. (a) Venography performed by direct puncture of the persistent lateral vein shows multiple perforators (arrowheads). (b) Diversion venography with application of a tourniquet (asterisk) was necessary to visualize the patent deep system (arrow). (c) The perforators were coil embolized prior to endovenous laser ablation the following day, taking care to oversize the coils by $30-50 \%$. CLOVES, congenital lipomatous overgrowth, vascular malformations, epidermal nevi, and spine/skeletal anomalies.
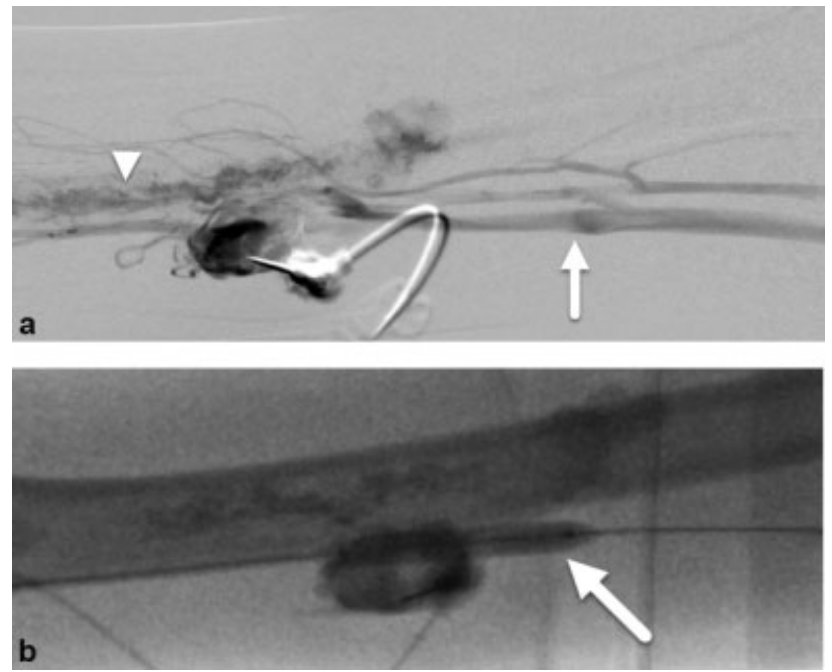

Fig. 6 (a) A 10-year-old male with a type 3 venous malformation (VM) extending into the bone, treated using a balloon occlusion technique. Direct injection of the right arm VM shows egress into the brachial vein (arrow), as well as communication into the medullary cavity of the humerus (arrowhead). (b) Placement of a $6 \mathrm{~mm} \times 4 \mathrm{~cm}$ balloon (arrow) across the communication via the peripheral brachial vein prevents outflow and allows for safe sclerosant injection.

to displace blood within the VM and expand within the lesion, making more extensive mural contact than liquid alone. The increased viscosity of a foamed sclerosant relative to its liquid counterpart also increases dwell time, resulting in prolonged duration of mural contact in the VM. Although foamed agents are also easily visible on US, it should be noted that foam will obscure visualization of deep tissues. It is therefore important to access and treat the deepest compo- nents of a malformation first, and then move progressively more superficially as needed.

Sodium tetradecyl sulfate (STS), polidocanol, and bleomycin may be injected in a foam formulation. As sclerotherapy is most often performed under fluoroscopy, a contrast agent may be added to the foam to increase conspicuity. Generation of foam relies on what is called the "Tessari method" or variations thereof, performed by repeatedly agitating a mixture of the sclerosant and air through a three-way connector and two syringes. Typically, a 1:4 mixture is used for polidocanol or STS, and foam has been described to be more stable when the connector hole is gradually narrowed, as the microbubbles become progressively smaller. ${ }^{26}$ Alternatively, a $0.2-\mu \mathrm{m}$ epidural filter (Perifix; B Braun, Bethlehem, PA) may be used for this purpose. These agents may also be foamed with watersoluble iodinated contrast to improve visualization under fluoroscopy and render the foam more stable. ${ }^{27}$ As both STS and polidocanol molecules consist of long hydrocarbon chains and hydrophilic heads, their foam can be rendered more stable with oily contrast (Lipiodol; Guerbet, Villepinte, France). It should be noted that adding contrast would reduce the concentration of the sclerosant in foam, and that oily contrast will add cost to the procedure. The typical formulation of foam in this setting is two parts of sclerosant, one part oily contrast, and two parts room air. There is no reported difference in efficacy between foaming of sclerosant with room air, or carbon dioxide $\left(\mathrm{CO}_{2}\right){ }^{28}$ Foam constituted with $\mathrm{CO}_{2}$ has a shorter half-life than that made from room air with faster absorption, and it may be favored in settings where a large volume of foam must be injected. Needles smaller than $27 \mathrm{G}$ are also thought to disrupt foam, and thus $25 \mathrm{G}$ or larger needles are recommended for injection. ${ }^{29}$ 


\section{Sclerotherapy Agents}

Systematic reviews have not found any particular agent to be superior for the treatment of vascular malformations, and this is thought to be due to limitations in study quality. ${ }^{30}$ The most commonly used sclerosant for VM is STS, and it has been shown to be cost-effective. ${ }^{30}$ STS is a detergent, and each molecule consists of a hydrophilic head and a long hydrophobic chain. STS is thought to act as a surfactant, inducing damage of the cell membrane by removal of transmembrane lipoproteins. STS is available in 1 and 3\% formulations (Sotradecol; Viatris, Pittsburgh, PA) and the $1 \%$ formulation may be used for smaller and more superficial lesions to reduce the risk of skin breakdown. The suggested dose limit for $3 \%$ STS is $0.5 \mathrm{~mL} / \mathrm{kg}$, but many practitioners limit the dose to 8 to $10 \mathrm{~mL}$ per session to control hemoglobinuria.

Polidocanol (also known as lauromacrogol 400) is also a detergent sclerosant that is commonly used to treat acquired venous varicosities, and is available as in liquid form in 0.5 and $1 \%$ concentrations (Asclera; Merz Aesthetics, Raleigh, $\mathrm{NC}$ ), and as a more expensive pre-made foam (Varithena; Boston Scientific, Marlborough, MA). Polidocanol is a milder agent than STS and is thought to have less efficacy in standard sclerotherapy settings. For vascular malformations, use is reserved for more superficial lesions or lesions where significant local inflammation needs to be avoided. Sodium morrhuate is another sclerosant, derived from bile salts of cod liver oil, which is sometimes used, although less commonly, in the United States. ${ }^{31}$

As discussed earlier, n-butyl cyanoacrylate (nBCA) glue (Histoacryl, B Braun; TruFill, Cordis, Santa Clara, CA) is used primarily to prevent outflow in VMs. Glue can be used as a primary sclerosant, as polymerization produces an exothermic reaction and a subsequent inflammatory foreign body response that is injurious to the target vessel endothelium. Glue is typically directly injected via a $21-G$ needle at a specific dilution (15-60\%) in oily contrast. The consistency should allow for a degree of desired penetration while avoiding nontarget embolization into deep veins. In general, glue should not be used in superficial locations, as it may leave a palpable lump with associated inflammatory reaction, or in nonsterile locations. This palpable nature, however, may facilitate surgical excision and in particular, intra-articular VMs may be embolized exclusively with glue and then excised by an orthopaedic surgeon ( - Fig. 7a-c). Glue is also provided in a high viscosity formulation with a catheter delivery system for closure of larger veins discussed later.

Bleomycin, a Streptomyces-derived antibiotic and chemotherapy agent frequently used for Hodgkin's lymphoma and germ cell tumors, has also shown efficacy for vascular malformations. ${ }^{32-34}$ It is chemically similar to pingyangmycin, which is used outside the United States, and is thought to act by oxidative damage to cells. It has also been found to interact with the mTOR pathway. ${ }^{35}$ For slow flow malformations, it is often used in locations where postprocedure inflammation needs to be limited, such as the oropharynx and the orbit. For VMs, foaming bleomycin with $25 \%$ human serum albumin is a particularly useful technique, and confers the benefits of foamed sclerosant described earlier, and allows for a greater volume of sclerosant to be given, as a per-session limit of $15 \mathrm{mg}$ or units $\left(15,000\right.$ international units) is commonly used. ${ }^{36}$ Idiosyncratic bleomycin-related complications (discussed in the section "Procedure-Related Complications") involve the lung and the skin, and are thought to be due to the relative absence of bleomycin hydrolase in these tissues. ${ }^{37}$ Important precautionary measures include decreasing $\mathrm{F}_{\mathrm{i}} \mathrm{O}_{2}$ during the procedure to room air and minimizing placement of adhesives on the skin.

Ethanol, provided in concentrations more than $95 \%$, acts by denaturation of proteins on contact, and is a very potent sclerosant. It is available as a liquid, in concentrations more
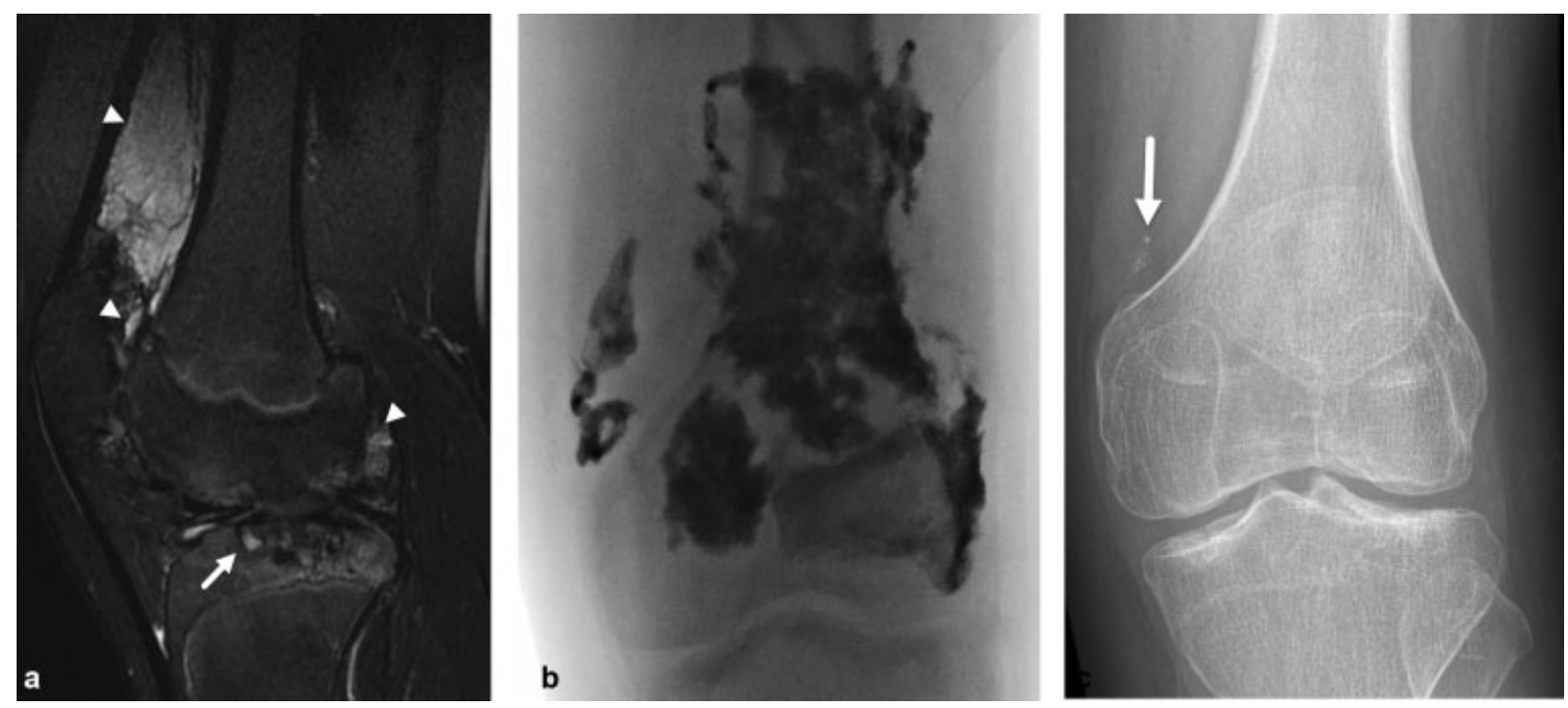

Fig. 7 A 16-year-old male with a right knee venous malformation and intra-articular involvement. (a) Sagittal T2 fat-saturated MRI demonstrating erosive change and subchondral cyst formation in the lateral tibia plateau (arrow) and an intra-articular venous malformation (arrowheads). (b) Frontal fluoroscopic image after sclerotherapy using 30\% n-butyl cyanoacrylate glue mixed with lipiodol shows intra-articular involvement. Excision was performed by orthopaedics the following day. (c) Follow-up radiograph obtained 14 months later shows only a small amount of radio-opaque glue remains (arrow) and healing of the degenerative changes of the tibia. 
than $95 \%$, or in a gel form outside the United States. The increased viscosity of gel may reduce the risk of nontarget embolization. ${ }^{38}$ It may be injected straight or mixed with oily or water-soluble contrast in a 3:1 dilution, to provide radioopacity. Due to its potency, ethanol is associated with increased rates of local tissue injury (skin and nerves) compared with bleomycin (and likely so compared with STS). ${ }^{34}$ As discussed earlier, careful assessment of lesion hemodynamics and vigilant injection of sclerosant must be performed to reduce these risks. High doses produce systemic contamination and can result in hemolysis, pulmonary vasospasm, and cardiovascular collapse. ${ }^{39}$ With regard to systemic complications, a dose limit of $0.1 \mathrm{~mL} / \mathrm{kg}$ every 10 minutes has been suggested safe, with a total dose per session under $1 \mathrm{~mL} / \mathrm{kg}^{40,41}$

\section{Endovenous Ablation}

Endovenous ablation, which includes laser ablation (EVLA), is reserved for closure of large malformed veins, and requires an intact deep venous system for physiologic drainage after closure. It is often used to close persistent embryonic veins in KTS, to address the risk of catastrophic PE. While most patients with KTS/PROS are thought to have intact deep systems, diversion venography may be required to document this prior to closure, if deep veins are not seen on MRI. ${ }^{13}$

EVLA is easier to perform in young children. Successful endovenous ablation usually requires closure of large confluences of the target vein-large tributaries and/or the central confluence-with coils or plugs, and this may necessitate a 2 -day procedure, with laser ablation performed on day 2 . These closures are easier in young children due to the smaller vein caliber. Laser ablation is also best reserved for the extrafascial component of the vein, as there is a risk of nerve injury for deeper segments that are poorly seen with US. EVLA requires tumescence around the vein to a thickness of 1 to $2 \mathrm{~cm}$, and this can be performed with $1 \%$ lidocaine with epinephrine $1: 100,000$ (the maximum dose is $7 \mathrm{mg} / \mathrm{kg}$; normal saline can be used after this limit is met). A minimum of $1 \mathrm{~cm}$ of separation of the vein from the skin should be achieved. We perform laser ablation using a 1,470-nm wavelength fiber (VenaCure system; Angiodynamics, Latham, NY), with an initial power of $10 \mathrm{~W}$ and an initial energy goal of $100 \mathrm{~J} / \mathrm{cm}$, beginning $2 \mathrm{~cm}$ proximal to the central confluence. The amount of energy deposition may have to be increased for larger diameter veins. ${ }^{42-45}$ Additional details for EVLA of VM are discussed in these cited references.

Newer modalities of endovenous ablations include a catheter-based viscous glue (VenaSeal; Medtronic, Minneapolis, MN) and pharmacomechanical device that employs motorized endovenous stripping (ClariVein; Merit, South Jordan, UT). ${ }^{45,46}$ Although the experience with these devices for VM is new, the potential advantages of these systems are the lack of thermal injury and decreased need for tumescence.

\section{Cryoablation}

Cryoablation has been used to ablate small residual AVM and vascular tumors such as FAVA and PTEN hamartoma, and is a promising modality for more focal VM. It is well-suited to treat focal intramuscular VM ( $\mathbf{- F i g . ~ 8 a - c ) . ~ A b l a t i o n ~ c a n ~ b e ~}$ performed with US or CT guidance, and typically involves two freeze periods separated by a 5- to 8-minute active thaw period. In general, the ice margin should extend at least $5 \mathrm{~mm}$ beyond the lesion. A target freeze period of 10 minutes is customary, but this may be reduced if adequate coverage of the iceball is seen, or there is concern about local soft-tissue injury (skin, nerve, or bowel). A separation of the skin surface and the ice margin of at least $5 \mathrm{~mm}$ is recommended to
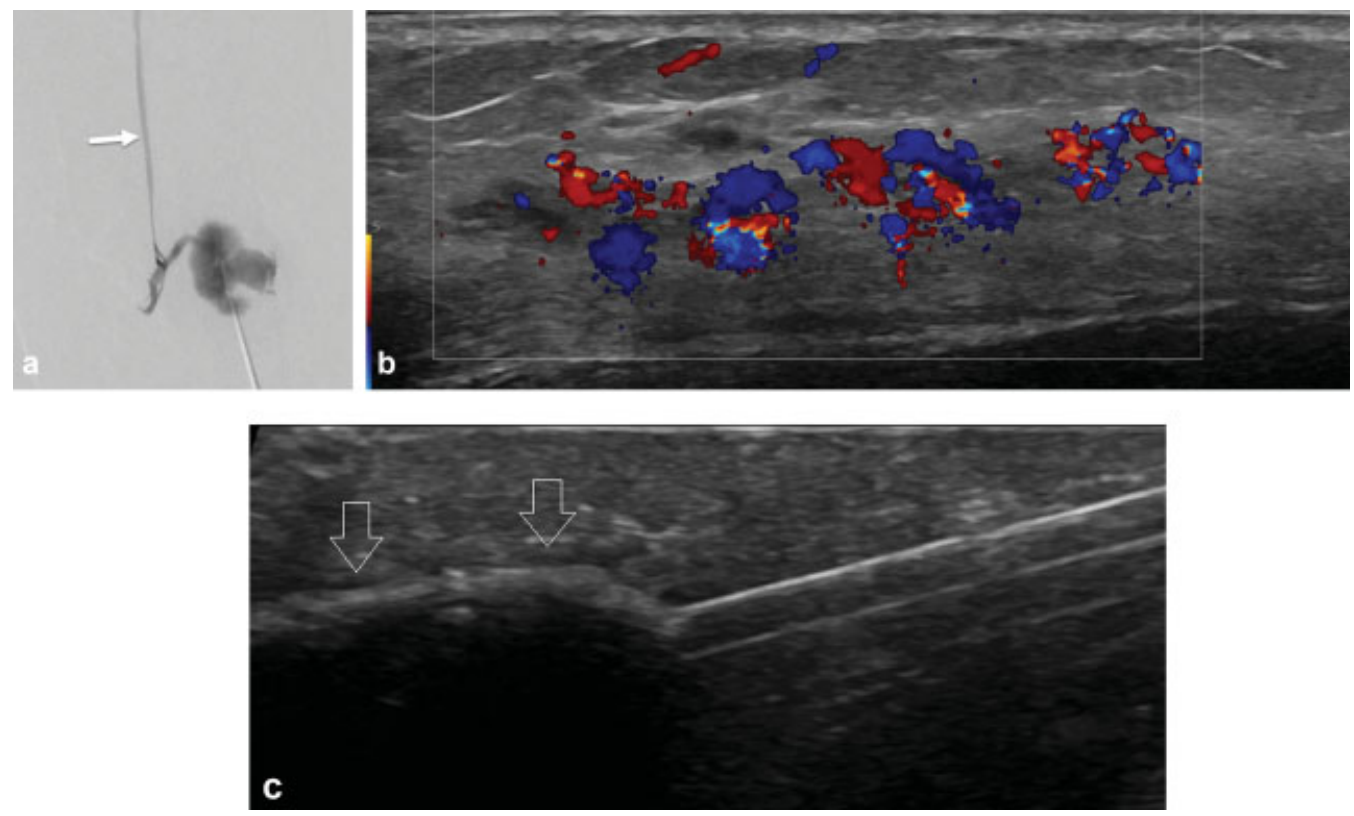

Fig. 8 A 10-year-old female with a painful shin type 2 venous malformation. (a) Fluoroscopic image obtained during direct injection shows egress into the saphenous vein (arrow). Outflow could not be controlled with direct compression or balloon occlusion; therefore, sclerotherapy was not performed. The lesion was treated with cryoablation. (b) Color Doppler US before and (c) grey scale US during shows adequate coverage with the ice ball (arrows). (Case courtesy of Dr. Anne Marie Cahill.) 
reduce the risk of frostbite. Adjunct techniques such as physiologic nerve monitoring for motor evoked potentials, temperature monitoring by thermocouple, or warm saline or $\mathrm{CO}_{2}$ fluid dissection may be useful. ${ }^{47-49}$ Due to pain caused by myositis, patients undergoing cryoablation of intramuscular extremity lesions may benefit from a nerve block for 1 to 2 days after the procedure.

\section{Surgical and Adjunct Methods}

Systematic reviews have not established surgery or sclerotherapy to be superior as standalone interventions, and primary extirpation of VM can result in significant morbidity and deformity. However, for certain lesions that are focal and/or respond poorly to sclerotherapy (e.g., glomuvenous malformation, blue rubber bleb nevus, or verrucous hemangioma), primary surgical resection is often the better option. Similarly, venous stripping can be performed for prominent superficial veins in lieu of endovenous ablation, as long as they do not have large connectors to the deep system. ${ }^{45}$

Surgery can be beneficial as an adjunct to percutaneous intervention. A common scenario for this is the excision of intraarticular or superficial VM after sclerotherapy or embolization with glue. For deeper lesions, glue may require excision if there is an intractable delayed hypersensitivity reaction (discussed later). Finally, external neodymium-doped yttrium aluminum garnet (Nd:YAG) laser therapy, typically performed by the plastic surgeon, may be useful in conjunction with sclerotherapy to treat superficial stigmata of VM. ${ }^{50}$

\section{Drug Therapy and Conservative Measures}

The elucidation of signal transduction defects in vascular anomalies-defects that produce dysregulated proliferation and angiogenesis-has resulted in a role for oncologic pharmacotherapy. Sirolimus, or rapamycin, which targets the mTOR pathway, is the index agent in this class. It is currently the primary medication available for VM, although direct PI3K inhibitor alpelisib may be an additional agent that targets this pathway (-Fig. 1). As the treatment duration is indefinite, and serum level titration requires frequent monitoring and dose adjustment, pharmacotherapy is reserved for lesions that are morbid and extensive, wherein the prognosis for lesion control by surgery and/or sclerotherapy is otherwise poor.

Conservative measures for control of symptoms from VM can be used in conjunction with any interventions, including oncologic pharmacotherapy. These are especially recommended for extensive malformations such as Bockenheimer or phlebectasia, and may include the use of anticoagulants (LMWH or DOAC, often prescribed through consultation with the hematologist) to control painful thrombus formation, and nonsteroidal analgesics when needed. ${ }^{19}$ Compression garments are also a mainstay of therapy for extensive lesions. Garments may be customized to the patient through consultation with the physical therapist; a pressure rating of 20 to $30 \mathrm{~mm} \mathrm{Hg}$ is commonly used for VM, but a pressure of $15 \mathrm{~mm}$ $\mathrm{Hg}$ may allow for greater comfort and compliance, with similar therapeutic benefit. ${ }^{51}$

\section{Procedure-Related Complications}

Site infection and blistering are the most common complications encountered in practice. Patients are advised to contact the interventional radiology team with concerns and submit photos via phone by SMS or e-mail. Skin blistering is common after STS sclerotherapy of superficial VM, and conservative wound care (nonadherent dressings with antibiotic ointment) is performed. If there is a concern for cellulitis, the patient is started on a 7-day course of oral antibiotic, frequently a firstgeneration cephalosporin (cefazolin) or clindamycin. Patients are advised to demarcate the erythema with a marker and follow progression or regression daily. If there is a poor response to oral antibiotics, the patient will come to the department for US assessment for abscess and possible admission for IV antibiotics.

For superficial blistering, supportive care and reassurance is continued until resolution. Skin breakdown indicates an inflammatory injury and presents as a large erosion and associated erythema. In the absence of infection, skin breakdown can be managed conservatively with wet-to-dry dressings and oral analgesics, with plastic surgery consultation for severe cases. The onset of skin necrosis is rapid, and it presents as black eschar (-Fig.9a). Plastic surgery consultation for possible skin graft is required.

Skin hyperpigmentation has been described in approximately $1 \%$ of patients treated with bleomycin and is often focal, and can be treated with topical hydroquinone 4 to $10 \%$ (-Fig. 9b). ${ }^{34}$ Diffuse and severe changes (flagellate) have been described even with doses of 15 units and may be intractable. ${ }^{52}$ Hyperpigmentation from bleomycin may be exacerbated by adhesives present on the skin during the procedure, and efforts are made to minimize the amount of adhesives (e.g., trimming electrocardiogram leads, using minimal paper tape for the eyes, and avoiding adhesive dressings). ${ }^{53}$ Focal hyperpigmentation has also been encountered with STS.

Bleomycin-related lung injury is a well-recognized complication. Pulmonary fibrosis is associated with total doses of 300 to 400 units, and has not yet been described with sclerotherapy. However, an acute pulmonary toxicity is also possible and is less well-known, and this may be catastrophic and result in respiratory failure or death. ${ }^{54,55}$ Fortunately, the incidence of this is rare, and is thought to be due to oxidative damage in alveoli with a poor capacity to metabolize bleomycin. ${ }^{37}$ Maintaining $\mathrm{F}_{\mathrm{i}} \mathrm{O}_{2}$ at room air during the procedure and postprocedure vigilance are advised.

Sclerotherapy-related nerve injury has been described with STS, but risks are likely higher with ethanol than with any other agent. ${ }^{34,56}$ Resulting neurologic deficits described in the literature have been self-limited, and motor deficits may be improved by physical therapy. Vigilant planning and injection are recommended to reduce this risk, with particular attention with VM in the head and neck. ${ }^{57}$

Glue boluses remain inert for most patients, but may rarely become infected and require antibiotics. Allergy to nBCA is rare, but may produce severe pruritus. Reported cases have been managed with oral and topical antihistamines. ${ }^{58}$ Chronically 

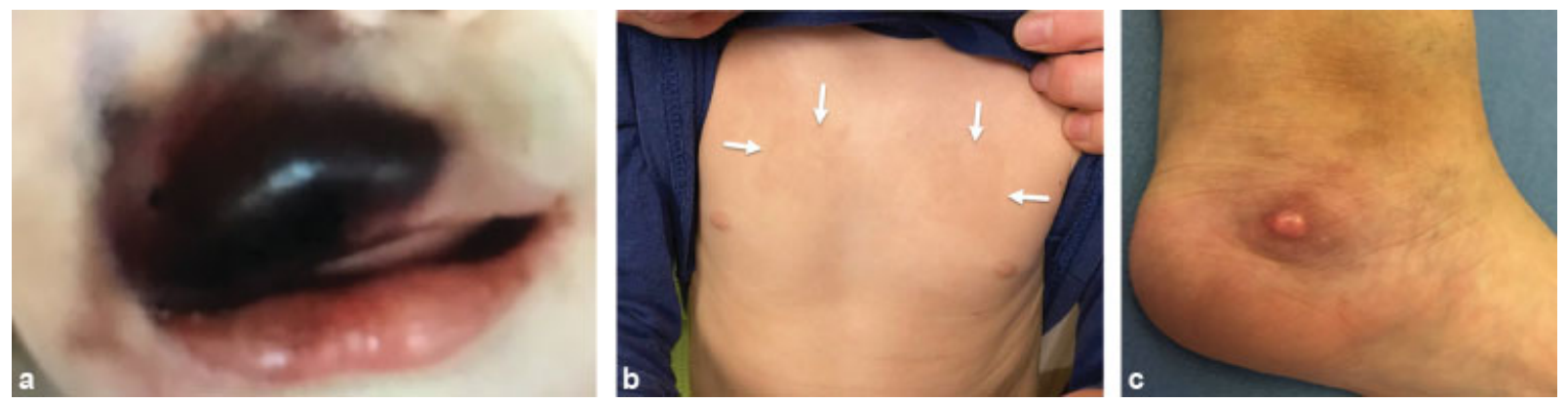

Fig. 9 Complications of sclerotherapy. (a) A 3-year-old with an upper lip venous malformation (VM) treated with STS foam. Photograph obtained shortly after treatment shows a dark eschar at the site of intervention from skin necrosis. The wound did not heal with conservative management and the patient required debridement and reconstruction by plastic surgery 3 weeks after treatment. (b) A 2-year-old with a biopsy-proven tufted angioma of the left upper arm treated with interstitial bleomycin injection. Photograph obtained 14 months after treatment shows square hyperpigmented foci (arrows) at the site of ECG lead placement. (c) A 14-year-old with a left heel VM treated by direction injection of $30 \%$ n-butyl cyanoacrylate. Photograph obtained 2 months later shows a small lump reflecting spontaneous glue extrusion. Once the glue was completely expelled, the site healed without further intervention.

infected or inflamed glue may eventually extrude through the skin (-Fig. 9c).

Glue or coils may also embolize to deep veins or the lungs during the procedure. Vigilant embolization with adequate outflow control is recommended to guard against this. Small volumes of glue pulmonary emboli are usually well-tolerated, but coils should be retrieved by snare whenever possible. If there is concern for deep venous injury and a risk of DVT, the patient is placed on LMWH twice per day or a DOAC for 2 weeks with US follow-up; if deep vein injury has occurred, anticoagulation will continue until follow-up in clinic in 6 to 8 weeks.

Extensive VM in the distal extremities (forearm or calf) may be prone to compartment syndrome after sclerotherapy. A preprocedure fascial release may be indicated for patients at high risk; otherwise overnight observation and expectant management are recommended.

As discussed previously, large VMs are often in a state of LIC due to their dysfunctional endothelium, and sclerotherapy can induce DIC. Rates of DIC in appropriately anticoagulated patients is low, but the patient should be assessed for signs and symptoms of DIC as appropriate. ${ }^{15,16,59}$ Hemoglobinuria is a noted complication of sclerotherapy with higher doses of STS and ethanol, but is usually transient and addressed by adequate hydration, unless excessive amounts are used. ${ }^{60}$

\section{Postprocedure Care}

Postprocedure care is directed toward control of pain and inflammation, and assessment for procedure-related complications discussed earlier. To reduce efflux of sclerosant from the lesion, a compression wrap should not be placed immediately after the procedure but is commenced after the postprocedure pain and inflammation subsides. Patients who undergo treatment of large lesions are admitted for observation and IV analgesia. Patients deemed appropriate for same-day discharge are given dressing supplies and instructions. They are prescribed oxycodone $(0.1 \mathrm{mg} / \mathrm{kg} /$ dose syrup every 6 hours for young children, and $5 \mathrm{mg}$ tablets every 6 hours for those older) for 3 to 5 days. Patients are encouraged to take acetaminophen between oxycodone doses, as needed. If a significant amount of pain and inflammation is anticipated, oral methylprednisolone as a 21-tablet dose pack, tapering over 6 days is also prescribed. Nonsteroidal anti-inflammatory drugs are usually avoided so as not to prohibit thrombosis. Elevation of the extremity and cold packs are applied as needed. A provider phones the patient the day after discharge to assess symptoms and any complications. Interventions that were planned in stages are usually separated by at least 4 weeks.

If the postprocedure course is adequately uneventful, the patient returns to interventional radiology clinic in 6 to 8 weeks for US and clinical assessment. The need for further intervention is then determined in this visit. Patient and provider expectations are also discussed.

\section{Conclusion}

VM is the most commonly encountered vascular anomaly in clinical practice. Understanding the pathogenesis, presentation, imaging findings, and complications are critical to making an accurate diagnosis and formulating a treatment plan. Clinical decision-making is best done in the multidisciplinary setting and treatment options should include conservative, medical, interventional, and surgical approaches. Interventional modalities are integral to the treatment of most patients.

Conflict of Interest

The authors have no conflicts of interest to disclose.

\section{References}

1 Wassef M, Blei F, Adams D, et al; ISSVA Board and Scientific Committee. Vascular anomalies classification: recommendations from the International Society for the Study of Vascular Anomalies. Pediatrics 2015;136(01):e203-e214

2 Cooke-Barber J, Kreimer S, Patel M, Dasgupta R, Jeng M. Venous malformations. Semin Pediatr Surg 2020;29(05):150976

3 Zúñiga-Castillo M, Teng CL, Teng JMC. Genetics of vascular malformation and therapeutic implications. Curr Opin Pediatr 2019;31(04):498-508 
4 Hage AN, Chick JFB, Srinivasa RN, et al. Treatment of venous malformations: the data, where we are, and how it is done. Tech Vasc Interv Radiol 2018;21(02):45-54

5 Fereydooni A, Dardik A, Nassiri N. Molecular changes associated with vascular malformations. J Vasc Surg 2019;70(01):314-326.e1

6 Marie A, Els C, Francine L. Pediatric vascular malformations: pathophysiology, diagnosis, and the role of interventional radiology. Cardiovasc Intervent Radiol 2011;34(04):691-704

7 Hassanein AH, Mulliken JB, Fishman SJ, Alomari AI, Zurakowski D, Greene AK. Venous malformation: risk of progression during childhood and adolescence. Ann Plast Surg 2012;68(02):198-201

8 Vogel SA, Hess CP, Dowd CF, et al. Early versus later presentations of venous malformations: where and why? Pediatr Dermatol 2013;30(05):534-540

9 Dalmonte P, Granata C, Fulcheri E, Vercellino N, Gregorio S, Magnano G. Intra-articular venous malformations of the knee. J Pediatr Orthop 2012;32(04):394-398

10 Reis J III, Alomari AI, Trenor CC III, et al. Pulmonary thromboembolic events in patients with congenital lipomatous overgrowth, vascular malformations, epidermal nevi, and spinal/skeletal abnormalities and Klippel-Trénaunay syndrome. J Vasc Surg Venous Lymphat Disord 2018;6(04):511-516

11 Esposito F, Ferrara D, Di Serafino M, et al. Classification and ultrasound findings of vascular anomalies in pediatric age: the essential. J Ultrasound 2019;22(01):13-25

12 Behravesh S, Yakes W, Gupta N, et al. Venous malformations: clinical diagnosis and treatment. Cardiovasc Diagn Ther 2016;6 (06):557-569

13 Alomari AI. Diversion venography-a modified technique in Klippel-Trenaunay syndrome: initial experience. J Vasc Interv Radiol 2010;21(05):685-689

14 Olivieri B, White CL, Restrepo R, McKeon B, Karakas SP, Lee EY. Low-flow vascular malformation pitfalls: from clinical examination to practical imaging evaluation - Part 2, venous malformation mimickers. AJR Am J Roentgenol 2016;206(05):952-962

15 Dompmartin A, Acher A, Thibon P, et al. Association of localized intravascular coagulopathy with venous malformations. Arch Dermatol 2008;144(07):873-877

16 Aronniemi J, Castrén E, Lappalainen K, et al. Sclerotherapy complications of peripheral venous malformations. Phlebology 2016; 31(10):712-722

17 Hung JWS, Leung MWY, Liu CSW, et al. Venous malformation and localized intravascular coagulopathy in children. Eur J Pediatr Surg 2017;27(02):181-184

18 Koo KSH, Dowd CF, Mathes EF, et al. MRI phenotypes of localized intravascular coagulopathy in venous malformations. Pediatr Radiol 2015;45(11):1690-1695

19 Mactier C, Mathias M, Drebes A. Novel approach to management of localised intravascular coagulopathy in extensive low flow venous malformations using rivaroxaban. Blood 2016;128(22): 3785

20 Correll D. The measurement of pain: objectifying the subjective. In: Waldman S, Bloch J, eds. Pain Management. W.B. Saunders; 2007:197-211

21 Varni JW, Seid M, Rode CA. The PedsQL: measurement model for the pediatric quality of life inventory. Med Care 1999;37(02): 126-139

22 Gurgacz S, Zamora L, Scott NA. Percutaneous sclerotherapy for vascular malformations: a systematic review. Ann Vasc Surg 2014;28(05):1335-1349

23 Chehab MA, Thakor AS, Tulin-Silver S, et al. Adult and pediatric antibiotic prophylaxis during vascular and IR procedures: a Society of Interventional Radiology Practice Parameter Update Endorsed by the Cardiovascular and Interventional Radiological Society of Europe and the Canadian Association for Interventional Radiology. J Vasc Interv Radiol 2018;29(11):1483-1501.e2

24 Puig S, Aref H, Brunelle F. Double-needle sclerotherapy of lymphangiomas and venous angiomas in children: a simple technique to prevent complications. AJR Am J Roentgenol 2003;180(05): 1399-1401

25 Legiehn GM. Sclerotherapy with adjunctive stasis of efflux (STASE) in venous malformations: techniques and strategies. Tech Vasc Interv Radiol 2019;22(04):100630

26 Tessari L, Cavezzi A, Frullini A. Preliminary experience with a new sclerosing foam in the treatment of varicose veins. Dermatol Surg 2001;27(01):58-60

27 Hashimoto K, Uchida B, Horikawa M, Mimura H, Farsad K. Effects of different mixing agents on the stability of sodium tetradecyl sulfate (STS) foam: an experimental study. Cardiovasc Intervent Radiol 2018;41(12):1952-1957

28 Peterson JD, Goldman MP. An investigation of side-effects and efficacy of foam-based sclerotherapy with carbon dioxide or room air in the treatment of reticular leg veins: a pilot study. Phlebology 2012;27(02):73-76

29 Cavezzi A, Tessari L. Foam sclerotherapy techniques: different gases and methods of preparation, catheter versus direct injection. Phlebology 2009;24(06):247-251

30 van der Vleuten CJM, Kater A, Wijnen MHWA, Schultze Kool LJ, Rovers MM. Effectiveness of sclerotherapy, surgery, and laser therapy in patients with venous malformations: a systematic review. Cardiovasc Intervent Radiol 2014;37(04):977-989

31 Zhao JH, Zhang WF, Zhao YF. Sclerotherapy of oral and facial venous malformations with use of pingyangmycin and/or sodium morrhuate. Int J Oral Maxillofac Surg 2004;33(05):463466

32 Muir T, Kirsten M, Fourie P, Dippenaar N, Ionescu GO. Intralesional bleomycin injection (IBI) treatment for haemangiomas and congenital vascular malformations. Pediatr Surg Int 2004;19(12): 766-773

33 Spence J, Krings T, terBrugge KG, da Costa LB, Agid R. Percutaneous sclerotherapy for facial venous malformations: subjective clinical and objective MR imaging follow-up results. AJNR Am J Neuroradiol 2010;31(05):955-960

34 Horbach SER, Rigter IM, Smitt JHS, Reekers JA, Spuls PI, van der Horst CMAM. Intralesional bleomycin injections for vascular malformations: a systematic review and meta-analysis. Plast Reconstr Surg 2016;137(01):244-256

35 Zhang W, Chen G, Ren JG, Zhao YF. Bleomycin induces endothelial mesenchymal transition through activation of mTOR pathway: a possible mechanism contributing to the sclerotherapy of venous malformations. Br J Pharmacol 2013;170(06):1210-1220

36 Ul Haq F, Mitchell SE, Tekes A, Weiss CR. Bleomycin foam treatment of venous malformations: a promising agent for effective treatment with minimal swelling. J Vasc Interv Radiol 2015; 26(10):1484-1493

37 Feldman D. Bleomycin-induced lung injury. 2020th ed. In: Kantoff PW, Freedman AS, Helen H, MF SD, eds. UpToDate; 2019. Accessed December 12, 2020 at: https://www.uptodate.com/contents/ bleomycin-induced-lung-injury/print

38 Teusch VI, Wohlgemuth WA, Hammer S, et al. Ethanol-gel sclerotherapy of venous malformations: effectiveness and safety. AJR Am J Roentgenol 2017;209(06):1390-1395

39 Hammer FD, Boon LM, Mathurin P, Vanwijck RR. Ethanol sclerotherapy of venous malformations: evaluation of systemic ethanol contamination. J Vasc Interv Radiol 2001;12(05):595-600

40 Yakes WF. Use of multiple sclerosant agents in vascular malformation management: a world in endovascular confusion and chaos. J Vasc Interv Radiol 2015;26(10):1494-1496

41 Shin BS, Do YS, Cho HS, et al. Effects of repeat bolus ethanol injections on cardiopulmonary hemodynamic changes during embolotherapy of arteriovenous malformations of the extremities. J Vasc Interv Radiol 2010;21(01):81-89

42 Fereydooni A, Nassiri N. Evaluation and management of the lateral marginal vein in Klippel-Trénaunay and other PIK3CA-related overgrowth syndromes. J Vasc Surg Venous Lymphat Disord 2020;8(03):482-493 
43 Patel PA, Barnacle AM, Stuart S, Amaral JG, John PR. Endovenous laser ablation therapy in children: applications and outcomes. Pediatr Radiol 2017;47(10):1353-1363

44 King K, Landrigan-Ossar M, Clemens R, Chaudry G, Alomari AI. The use of endovenous laser treatment in toddlers. J Vasc Interv Radiol 2013;24(06):855-858

45 Huegel U, Baumgartner I. Implementation of new endovenous treatments in therapy for lateral embryonic veins. J Vasc Surg Cases Innov Tech 2019;5(03):243-247

46 Lambert G, Teplisky D, Cabezas M, et al. Mechanochemical endovenous ablation of varicose veins in pediatric patients with Klippel-Trénaunay syndrome: feasibility, safety, and initial results. J Vasc Interv Radiol 2021;32(01):80-86

47 Carabin J, Bouhamama A, Vaz G, et al. Percutaneous cryoablation of symptomatic intramuscular venous malformation. J Vasc Interv Radiol 2020;31(04):558-563.e3

48 Shaikh R. Percutaneous image-guided cryoablation in vascular anomalies. Semin Intervent Radiol 2017;34(03):280-287

49 Ramaswamy RS, Tiwari T, Darcy MD, et al. Cryoablation of low-flow vascular malformations. Diagn Interv Radiol 2019;25(03):225-230

50 Gregory S, Burrows PE, Ellinas H, Stadler M, Chun RH. Combined $\mathrm{Nd}$ :YAG laser and bleomycin sclerotherapy under the same anesthesia for cervicofacial venous malformations: a safe and effective treatment option. Int J Pediatr Otorhinolaryngol 2018; 108(108):30-34

51 Rabe E, Partsch H, Hafner J, et al. Indications for medical compression stockings in venous and lymphatic disorders: an evidence-based consensus statement. Phlebology 2018;33(03): 163-184
52 Abess A, Keel DM, Graham BS. Flagellate hyperpigmentation following intralesional bleomycin treatment of verruca plantaris. Arch Dermatol 2003;139(03):337-339

53 Milbar HC, Jeon H, Ward MA, Mitchell SE, Weiss CR, Cohen BA. Hyperpigmentation after foamed bleomycin sclerotherapy for vascular malformations. J Vasc Interv Radiol 2019;30(09):1438-1442

54 Méndez-Echevarría A, Fernandez-Prieto A, de la Serna O, et al. Acute lung toxicity after intralesional bleomycin sclerotherapy. Pediatrics 2018;141(01):e20161787

55 Atwa K, Abuhasna S, Shihab Z, Hashaykeh N, Hasan R. Acute pulmonary toxicity following intralesional administration of bleomycin for a lymphovenous malformation. Pediatr Pulmonol 2010;45(02):192-196

56 Stuart S, Barnacle AM, Smith G, Pitt M, Roebuck DJ. Neuropathy after sodium tetradecyl sulfate sclerotherapy of venous malformations in children. Radiology 2015;274(03):897-905

$57 \mathrm{Hu} \mathrm{X}$, Chen D, Jiang C, et al. Retrospective analysis of facial paralysis caused by ethanol sclerotherapy for facial venous malformation. Head Neck 2011;33(11):1616-1621

58 Sinha KR, Duckwiler G, Rootman DB. Urticarial reaction following endovascular embolization of an orbital arteriovenous malformation (AVM) with n-butyl cyanoacrylate (nBCA) glue. Interv Neuroradiol 2017;23(06):666-668

59 Greene AK, Alomari AI. Management of venous malformations. Clin Plast Surg 2011;38(01):83-93

60 Barranco-Pons R, Burrows PE, Ossar-Landrigan M, et al. Gross hemoglobinuria and oliguria are common transient complications of sclerotherapy for venous malformations: review of 475 procedures. Am J Roentgenol 2012;199(03):691-694 